\title{
Abjektion und existenzielle Krise
}

\author{
Tobias Schramm
}

Eingegangen: 1. Juni 2021 / Angenommen: 27. Oktober 2021 / Online publiziert: 26. November 2021

(C) Der/die Autor(en) 2021

Zusammenfassung Julia Kristevas Konzept der Abjektion, das sie in ihrer Monografie Powers of Horror. An Essay on Abjection (1982) vorstellt, wird in der Rezeption nicht selten mit Prozessen des Ausschlusses und der Exklusion gleichgesetzt - sei es auf innerpsychischer oder sozialer Ebene. Das ist zunächst nicht verwunderlich, spielt doch die Grenze und Grenzziehung in ihrem Werk eine entscheidende Rolle: Die Abjektion ist die Grundlage der Konstitution des Subjektes und damit auch der kulturellen Ordnung, die Subjekte hervorbringt. Nur durch die Grenzziehung, als Produkt der Abjektion, können Subjekte eine stabile Psyche und eine kulturelle Identität überhaupt erst entwickeln.

Diese Lesart verkennt jedoch die grundlegende theoretische Anlage des Konzeptes bzw. wendet den Begriff bereits auf psychische, aber auch soziale Prozesse an, für welche die Abjektion zunächst erst einmal die Grundlage bildet: Bei der Abjektion, so meine Lesart des Begriffs, handelt es sich primär um die Herstellung einer Grenze. Erst die Herstellung einer Grenze bildet schließlich die Grundlage für Prozesse des Ausschlusses, und zwar sowohl in der frühkindlichen Subjektbildung als auch in der Etablierung kultureller Grenzziehungen und damit der Konstitution sozialer Ordnung. Das Erleben des Abjekten ist, so argumentiere ich in diesem Beitrag, im Grunde gleichzusetzen mit einer existenziellen Krise: Durch die Irritation ontologischer Grenzen findet sich das Subjekt in einem Zustand existenzieller Angst wieder.

So bieten zahlreiche Ansätze wie etwa Kant, Kierkegaard oder Heidegger in ihren Begriffsinstrumentarien differenzierte Konzepte der, existenziellen Angst ' bzw. einer existenziellen Krise an, die es ermöglichen, den speziellen psychologischen Zustand, den Kristeva mit dem Begriff des Abjekts adressiert, als existenzielle Angst oder Krise (bzw. als existenzielle Ungewissheit) zu verstehen.

Tobias Schramm $(\bowtie)$

Friedrich-Alexander-Universität Erlangen-Nürnberg, Erlangen, Deutschland

E-Mail: tobias.schramm@fau.de 
Schlüsselwörter Kristeva · Grenzziehungen · Krise des Subjekts · Ungewissheit · Existenzielle Angst

\section{Abjection and existential crisis}

Abstract The concept of abjection, which was famously introduced by Julia Kristeva in Powers of Horror. An Essay on Abjection (1982), is quite often referred to the phenomena or the process of exclusion-either forms of psychological or social exclusion. This is not very surprising: the concept of boundaries and the process of setting these boundaries play an important role in Powers of Horror. The process of abjection is the foundation for subjectivity and therefore the fundamental basis for culture and the possibility to form cultural identities. Only through abjection and the establishing of fundamental boundaries is it possible to become a subject-as well as a subject of a certain cultural system. But this is not the way I want to read Kristeva, as it misunderstands the theoretical groundworks of the concepts of abject and abjection. This way of understanding the concept of abjection identifies the process of exclusion as abjection. I want to argue that abjection is not to be identified as exclusion but rather to be understood as a precondition of exclusion. And I want to clear out a second misconception of Kristevas' work: that the concept of abject is not to be understood as an object. Instead, abject is rather to be referred to as a state of mind, best explained in ways of Kants' or Kierkegaards' and Heideggers' (1979 [1927], 2006 [1929]) concept of existential fear.

Keywords Kristeva $\cdot$ Crisis of the subject $\cdot$ Boundaries $\cdot$ Uncertainty $\cdot$ Existential fear

It is thus not lack of cleanliness or health that caused abjection but what disturbs identity, system, order. What does not respect borders, position, rules. The in-between, the ambiguous, the composite (Kristeva 1982, p. 4).

\section{Einleitung: Grenzziehungen}

Julia Kristevas Konzept der Abjektion, das sie in ihrer Monografie Powers of Horror. An Essay on Abjection (1982) vorstellt, wird in der Rezeption nicht selten mit Prozessen des Ausschlusses und der Exklusion gleichgesetzt - sei es auf innerpsychischer oder sozialer Ebene (vgl. dazu Butler 1993 oder Arya 2014). Das ist zunächst nicht verwunderlich, spielt doch die Grenze und Grenzziehung in ihrem Werk eine entscheidende Rolle: Die Abjektion ist die Grundlage der Konstitution des Subjektes und damit auch der kulturellen Ordnung, die Subjekte hervorbringt. Nur durch die Grenzziehung, als Produkt der Abjektion, können Subjekte eine stabile Psyche und eine kulturelle Identität überhaupt erst entwickeln.

Diese Lesart verkennt jedoch die grundlegende theoretische Anlage des Konzeptes bzw. wendet den Begriff bereits auf psychische, aber auch soziale Prozesse an, 
für welche die Abjektion zunächst erst einmal die Grundlage bildet ${ }^{1}$ : Bei der $\mathrm{Ab}$ jektion, so meine Lesart des Begriffs, handelt es sich primär um die Herstellung einer Grenze. Erst die Herstellung einer Grenze bildet schließlich die Grundlage für Prozesse des Ausschlusses, und zwar sowohl in der frühkindlichen Subjektbildung als auch in der Etablierung kultureller Grenzziehungen und damit der Konstitution sozialer Ordnung. Das Erleben des Abjekten ist, so argumentiere ich in diesem Beitrag, im Grunde gleichzusetzen mit einer existenziellen Krise: Durch die Irritation ontologischer Grenzen findet sich das Subjekt in einem Zustand existenzieller Angst wieder. Und die existenzielle Krise des Subjekts muss durch Abjektion, der (Wieder-)Herstellung dieser Grenzen, behoben werden.

Ich möchte insofern den Begriff der existenziellen Krise als Ausgangspunkt dafür nutzen, um die Begriffe der Abjektion und des Abjekten verständlich und die Theorie Kristevas philosophisch anschlussfähig zu machen - auch abseits einer psychoanalytischen oder sozialwissenschaftlichen Rezeption hinaus. So bieten zahlreiche Ansätze wie etwa Kant (1786), Kierkegaard (1992) oder Heidegger (1979 [1927], 2006 [1929]) in ihren Begriffsinstrumentarien differenzierte Konzepte der ,existenziellen Angst' bzw. einer existenziellen Krise an, die es ermöglichen, den speziellen psychologischen Zustand, den Kristeva mit dem Begriff des Abjekts adressiert, als existenzielle Angst oder Krise (bzw. als existenzielle Ungewissheit) zu verstehen.

Im Folgenden werde ich daher zunächst in das Konzept der existenziellen Krise einführen und besonders auf das auch für neuere Ansätze grundlegende Konzept der Ungewissheit eingehen, das bereits bei Immanuel Kant angelegt ist. Die Überwindung dieser Krise, so Kant, beruht basal auf der Fähigkeit, eine reflexive Distanz zum eigenen Begehren aufzubauen und eine Grenze, die durch die Vernunft bestimmt wird, einzuführen. Mithilfe des Begriffs der existenziellen Krise will ich zeigen, dass Abjektion als Herstellung von der eigenen Subjektivität in einem metaphysisch-psychologischen Sinn handelt - nämlich der Herstellung ontologischer Grenzen des Subjekts.

Die Abjektion ist damit nicht der Prozess des Ausschlusses, sondern wird als Herstellung einer Grenze verstehbar, die einen Ausschluss erst möglich macht. Und das Abjekt ist damit nicht als empirisches Objekt, welches ausgeschlossen wird, sondern als Zustand der Ungewissheit zu verstehen. ${ }^{2}$ Abschließend wird dafür plädiert, auch umgekehrt den analytischen Mehrwert der Theorie Kristevas für die Soziologie und Philosophie nutzbar zu machen: Und damit sind insbesondere Theorien gemeint, die Begriffe der Ungewissheit (oder Gewissheit) und Selbstschöpfung bearbeiten.

\section{Existenzielle Ungewissheit, oder: Die Vertreibung aus dem Paradies}

In seinem Aufsatz mit dem Titel Mutmaßlicher Anfang der Menschengeschichte stellt Kant (1786) den Menschen, als, wie es die philosophische Anthropologie - so

\footnotetext{
1 So wird nicht selten Judith Butlers Verwendungsweise des Begriffs der Abjektion herangezogen, wenn ein sozialer oder kultureller Ausschluss erklärt werden soll (vgl. dazu Butler 1993). Und dies obwohl Butlers Verwendungsweise der Begriffe des Abjekt und der Abjektion von Kristeva deutlich abweicht.

2 Im Sinne von Wittgensteins’ Auffassung von Gewissheit (vgl. dazu Wittgenstein 1984).
} 
beispielsweise Plessner oder Gehlen - (vgl. Fischer 2016) beschreiben würde, in die existenzielle Ungewissheit eines instinktreduzierten und weltoffenen Wesens geworfen vor. Das Beispiel, das Kant hierfür wählt, ist in keinerlei Hinsicht trivial und dazu metaphysisch stark aufgeladen: der Sündenfall als Urszene der menschlichen Subjektwerdung.

Der Mensch im Garten Eden ist, so interpretiert es Kant (1786, S. 111), zunächst allein von seinen Instinkten geleitet. Diese geben dem Menschen alle notwendigen Regeln für das Leben im Paradies vor. Durch seinen Instinkt erhält der Mensch ein erstes normatives Prinzip: Er bestimmt, wie er sich in welchen Situationen verhält, was er begehrt und was er fürchtet und was er isst. Aber zur ersten normativen Ausstattung des Menschen gehört nicht nur der Instinkt, sondern auch die Vernunft. Und so stellt der Mensch mittels seiner Vernunft auch Überlegungen über die Objekte seiner Ernährung an und damit auch über die Objekte, die zur Befriedigung seiner Bedürfnisse geeignet sind. Kant spekuliert darüber, dass einerseits der Apfel mit einer Birne verglichen worden sei - „Birnen schmecken gut, der Apfel sieht aus wie eine Birne, also sollten wir doch mal den Apfel probieren“. Oder aber: „Die Schlange ist ein Lebewesen wie wir, und sie isst den Apfel; also sollten wir doch mal den Apfel probieren“ (vgl. dazu Korsgaard 2009, S. 117-118). Der Vergleich führt dazu, dass der Mensch zum ersten Mal vor einer Entscheidung steht:

[S]o konnte dieses schon der Vernunft die erste Veranlassung geben, mit der Stimme der Natur zu chikaniren (III, 1) und trotz ihrem Widerspruch, den ersten Versuch von einer freien Wahl zu machen, der als der erste wahrscheinlicherweise nicht der Erwartung gemäß ausfiel (Kant 1786, S. 112).

Im Zuge der ersten Entscheidung setzt sich der Mensch durch den Vergleich ein eigenes normatives Prinzip: ich sollte den Apfel essen, um meinen Hunger zu stillen. Die Entscheidung, dieser Maxime nachzugehen, wird die erste Instanz der Willensfreiheit des Menschen. Er erkennt sich von seinen natürlichen Trieben geleitet. Aber durch den Vergleich und die Tätigkeit der Vernunft eröffnet er sich selbst die Möglichkeit, ein Objekt, das nicht von seinen Instinkten bestimmt wird, auszuwählen, um seinen Hunger zu stillen. In der konkreten Auswahl des Objekts, des Apfels folgt er nicht seinem Instinkt, sondern seiner Fähigkeit, vernünftig zu sein und Entscheidungen zu treffen:

Aus dieser Darstellung der ersten Menschengeschichte ergibt sich: da der Ausgang des Menschen aus dem ihm durch die Vernunft, als erster Aufenthalt seiner Gattung vorgestellten Paradieses nicht anders, als der Übergang aus der Rohigkeit eines bloß thierischen Geschöpfes in die Menschheit, aus dem Gängelwagen des Instinkts zur Leitung der Vernunft, mit einem Worte, aus der Vormundschaft der Natur in den Stand der Freiheit gewesen sei (Kant 1786, S. 115).

Indem er sich selbst normative Prinzipien gibt, die sein Handeln bestimmen, macht sich der Mensch, so Kant, zum Urheber seiner selbst. Er „befreit“ sich in der ersten freien Entscheidung (die übrigens Eva zugeschrieben werden kann) von der Handlungsdetermination durch seine Instinkte. Das heißt aber nicht, dass Instinkte nicht mehr weiter wirksam sind (vgl. dazu Kant 1786, S. 112-115), doch schafft, 
so Kant, das menschliche Bewusstsein die Bedingungen der Möglichkeit einer kritischen Distanz zu den Instinkten und damit einen Raum, in dem diese Instinkte bewusst werden können. Der Mensch steht zu sich selbst also in einer bestimmten Art von reflexiver Distanz (vgl. dazu Kant 1786, Frankfurt 2001; Korsgaard 2009). Diese reflexive Distanz ermöglicht es ihm, sich eigene Handlungsprinzipien zu geben, und sich von seinen Trieben zu distanzieren: „Wo Es war, soll Ich werden“ (Freud 1933, S. 86).

Qua der Distanz durch sein Bewusstsein wird es dem menschlichen Subjekt möglich, nicht mehr rein durch die biologischen Instinkte bestimmt zu sein - nicht dadurch, dass alle biologischen Anreize und Instinkte aus seinem Wesen getilgt werden. Dies wäre auch nicht möglich. Aber das menschliche Subjekt kann bestimmen, wann und wie es seinen Trieben und Begierden folgen soll.

Aber mit der Lösung von den Instinkten geht nach Kant auch ein großer Verlust einher. Der Mensch verliert die durch Instinkte wohlstrukturierte Welt, in der die Bedeutung der Objekte, also was sie sind und welchen Zweck sie haben, vorgegeben war. Ohne diese Instinkte ist der Mensch in ein ununterschiedenes Chaos geworfen. Er ist mit Ungewissheit konfrontiert und erfährt eine existenzielle Krise:

Er entdeckte in sich ein Vermögen, sich selbst eine Lebensweise auszuwählen, und nicht gleich anderen Thieren an eine einzige gebunden zu sein. Auf das augenblickliche Wohlgefallen, das ihm dieser bemerkte Vorzug erwecken mochte, mußte doch sofort Angst und Bangigkeit folgen: wie er, der noch kein Ding nach seinen verborgenen Eigenschaften und entfernten Wirkungen kannte, mit seinem neu entdeckten Vermögen zu Werke gehen sollte. Er stand gleichsam am Rande eines Abgrundes; denn aus einzelnen Gegenständen seiner Begierde, die ihm bisher der Instinct angewiesen hatte, war ihm eine Unendlichkeit derselben eröffnet, in deren Wahl er sich noch gar nicht zu finden wußte (Kant 1786, S. 112).

Die Fähigkeit, eine eigene Lebensweise auszuwählen (der Sündenfall) geht damit mit dem Erwachen unserer Vernunft und damit mit dem Ausschluss aus dem Paradies einher. Der Mensch muss also nicht nur seine eigene Subjektivität, sondern auch die Welt, in der er lebt, selbst schöpfen. Er ist jedoch zunächst gezwungen, sich mit der Ungewissheit und Angst auseinanderzusetzen, was er tun soll, was er selbst ist und was die Dinge der Welt für ihn bedeuten und welchen Zweck sie haben sollen. Und Kant ist der erste Philosoph, der explizit diese existenzielle Angst und Ungewissheit thematisiert (ihm folgen dann aber in der Problematisierung dieser existenziellen Angst unter anderem Kierkegaard und Heidegger) ${ }^{3}$. Der Ausschluss aus dem Paradies ist damit nicht nur eine Geschichte der Willensfreiheit und der Geburt des selbstbestimmten und vernunftbegabten Menschen. Es ist auch die Geschichte eines Abgrundes, eine Geschichte von existenzieller Angst und Schrecken. Und es ist diese existenzielle Krise der Ungewissheit, in welcher sich der psychische Zustand des Abjekten verorten lässt.

\footnotetext{
3 So beschreibt beispielsweise Kierkegaard (1992) in Der Begriff Angst die erste freie Entscheidung - die erste freiheitliche Selbstsetzung bzw. die Setzung des ersten normativen Prinzips im menschlichen Sündenfall - im Anschluss an Kant als fehlgeleitete Selbstsetzung.
} 


\section{Die Schrecken und Möglichkeiten der Ungewissheit: Abjekt und Abjektion}

Durch die Herstellung einer Grenze konstituieren wir uns, nach Kristeva, als Subjekt - und damit auch als kulturelles Subjekt, da bestimmte Grenzziehungen nur in einem bestimmten kulturellen Rahmen geboten sind. Die Grenzziehung ist Teil unserer ontologischen Realität - einmal, um uns generell als Subjekt etablieren und verstehen zu können und andererseits, um uns immer wieder der Gewissheit einer bestimmten kulturellen Existenz zu versichern. Wir vergewissern uns unserer individuellen, aber auch sozialen und kulturellen Identität: „There, abject and abjection are my safeguards. The primers of my culture“" (Kristeva 1982, S. 2). Die Abjektion, die auf die Irritation einer Grenze reagiert, konstituiert und sichert somit nicht nur grundlegende kulturelle Normen, sondern auch unsere Möglichkeit, sich innerhalb der Welt zurechtfinden zu können. Sie sichert unseren Standpunkt in der Welt (Kristeva 1982; Taylor 1996).

\subsection{Abjekt als Zustand der Ungewissheit}

Ähnlich wie Kant den Zustand des in die Welt geworfenen Subjekts als einen durch „Angst und Bangigkeit“ bestimmten Zustand ,am Rande eines Abgrundes“ (Kant 1786, S. 112) bestimmt, beschreibt Julia Kristeva das Abjekt in Powers of Horror als die Erfahrung der „,emptiness“ (Kristeva 1982, S. 6) als das Gefühl: „,nothing is familiar, not even the shadow of a memory“ (Kristeva 1982, S. 6).

Abjekt ist also nicht, wie es oft verstanden wird, ein bestimmtes Objekt, das etwa mit Ekel besetzt ist (siehe dazu bspw. Menninghaus 1999) und von dem sich das Subjekt radikal abwenden muss, sondern die existenzielle Bedrohung des Subjekts und seiner Welt durch die Auflösung ontologischer Sicherheiten (also epistemischer Grenzen). Es ist stattdessen die Auflösung aller Prinzipien, sich in der Welt zu orientieren: Ein Objekt ist immer nur ein Objekt, weil es bestimmte Kriterien gibt, es zu bestimmen und sich ihm gegenüber zu verhalten. Ein Subjekt ist nur Subjekt, wenn es Kriterien hat, die Entscheidungen und Handlungen ermöglichen: also Handlungsprinzipien im kantischen Sinne (Kant 1986):

Wenn wir in Gedanken alle Begrenzungen aufheben, so bleibt nicht ein Wille übrig, der kraft seiner Unbegrenztheit besonders große Freiheit besitzt. Was übrig bleibt, ist, weil es kein bestimmter Wille mehr ist, überhaupt kein Wille mehr (Bieri 2007, S. 240).

Der Begriff des Abjekts bezeichnet genau diese Abwesenheit von Kriterien und die Ungewissheit und existenzielle Angst und damit Krise eines menschlichen Subjekts. Gleichzeitig ist die Abjektion der erste Moment einer Entscheidung, also des Setzens von Grenzen und Kategorien. Doch zunächst fehlen diese Grenzen und das Abjekt ist nichts, worauf wir Bezug nehmen können: „When I am beset by abjection, the twisted braid of affects and thoughts I call by such a name does not have, properly speaking, a definable object" (Kristeva 1982, S. 1; Herv. i. Orig.). Abjekt ist kein empirisches Objekt, sondern das Fehlen einer Ordnung, die Abwesenheit klarer Grenzen und die Unfähigkeit des Subjekts, eine klare Grenzziehung vorzu- 
nehmen: „The abject has only one quality of the object - that of beeing opposed to I“ (Kristeva 1982, S. 1; Herv. i. Orig.). Stattdessen besteht der Zustand des Abjekten allein in der Ungewissheit. Er zeigt sich in der Angst und dem Schrecken der Unsicherheit, nicht unterscheiden zu können, was Teil von uns und was nicht mehr Teil von uns selbst ist. Insofern ist es eine phänomenologische Erscheinung bzw. die Form fehlender Differenzierung. Der Zustand des Abjekten schafft insofern einen Raum, in dem Bedeutungen aufhören zu existieren: ,[W] hat is abject, [...] draws me toward the place where meaning collapses" (Kristeva 1982, S. 2). Grenzen werden nicht einfach nur überschritten, sondern gänzlich aufgelöst. Der Zustand des Abjekten zeigt sich nur in der Abwesenheit bzw. Ungewissheit einer klaren Grenze - in der Unmöglichkeit Subjekt und Objekt, Innen und Außen, Eigenes und Fremdes zu trennen.

Und damit können wir eine entscheidende Analogie zu Kants mutmaßlichem Beginn der Menschheit ziehen. Wie zuvor dargelegt, ist es die Fähigkeit zur Willensfreiheit, die Fähigkeit des Menschen, eine reflexive Distanz zu seinen Begierden einzunehmen, welche die naturgegebene Ordnung der Welt für das Subjekt auflöst. Kristeva beschreibt ebenfalls einen solchen Verlust der Struktur der Welt durch das Abjekt und damit die Aufgabe, sich selbst zu schöpfen: ,[A]ll its objects [die Objekte des Subjekts, Anm. d. Autors] are based merely on the inaugural loss that laid the foundations of its own“ (Kristeva 1982, S. 5, Herv. i. Orig.). Der Mensch ist in dieser Hinsicht nicht nur zu Freiheit verdammt, sondern auch dazu, seine Welt sinnhaft zu strukturieren. Die reflexive Distanz des Menschen, die ihn aus der Welt der Instinkte herauswirft und damit vor die Aufgabe stellt, eine Welt und die Objekte dieser Welt selbst zu bestimmen, ist die Geburt des Zustands des Abjekten. Abjekt, bzw. der phänomenologische Zustand der Ungewissheit - die Schnittstelle zwischen Unordnung der Welt und Willensfreiheit - ist ein Zustand, der das Subjekt zwingt, sich selbst zu schöpfen, indem es eine Grenze, eine Unterscheidung etabliert. Und genau diesen Vorgang der Selbstgesetzgebung beschreibt Kristeva mit der Abjektion und damit mit der Einführung einer Grenze.

\subsection{Abjektion und die menschliche (Selbst-)Schöpfung}

Um das Phänomen der Abjektion besser verstehen zu können, muss man sich das Subjekt und dessen Identität als Prozess vorstellen. Das Subjekt bzw. das „Ich“ ist kein festes bzw. festgelegtes Wesen und keine abgetrennte, unbeeinflussbare Entität, sondern immer abhängig von der Abgrenzung von anderen Objekten und Subjekten (Hegel 1986). Das Gefühl der eigenen Subjektivität und des eigenen „Ichs“ wird insofern durch die Abgrenzung bzw. Abtrennung von Objekten und durch die Positionierung in einem sozialen Raum mit und durch andere Subjekte überhaupt erst hergestellt (vgl. dazu Taylor 1983, 1996). Subjektivität ist das Produkt eines Prozesses von Grenzziehungen und der Übernahme von Grenzen und Unterscheidungen, die bereits durch eine soziale oder kulturelle Ordnung hergestellt worden sind.

Was genau das Subjekt gegen die Bedrohung der Auflösung oder Überschreitung individueller und sozialer Grenzen bewahrt, adressiert Kristeva mit dem Konzept der Abjektion. 
Die Abjektion ist damit die Antwort - entweder des psychischen Subjekts oder des sozialen Subjekts - auf die Bedrohung oder Auflösung einer Grenze; dass beispielsweise der Körper nicht einfach nur „,menschlich“, sondern auch animalisch sein kann. Der kreatürliche Teil des menschlichen Selbst stellt insofern ständig das individuelle und soziale Selbstverständnis und die kulturellen Grenzziehungen in Frage. Die Folge ist, dass Subjekte versuchen müssen, diejenigen Objekte, die eine Irritation oder Auflösung der Grenzen hervorrufen, aus ihrer Welt auszuschließen.

In Kants Urszene der existenziellen Krise haben wir gesehen, dass der Mensch durch sein Bewusstsein und seine Vernunft dazu in der Lage ist, seine natürlichen Begierden zu hinterfragen. Diese Analogie kann genutzt werden, um zu verstehen, warum für Kristeva das Abjekt insbesondere in der Konfrontation des Menschen mit seiner auch schwer zu kontrollierenden Kreatürlichkeit hervortritt: Zwar können sich Subjekte von ihren Instinkten distanzieren, aber sie sind dennoch immer Teil des eigenen Selbst. Sie werden sie nie endgültig überwinden können und sind stetig vor die Aufgabe gestellt, zu entscheiden, inwiefern sie ihren Instinkten Macht über sich selbst zugestehen. Als Wesen, das dazu fähig ist, sich selbst zu schöpfen, ist der Mensch ständig zerrissen. Das Abjekt findet sich aber nicht in der ständigen Erinnerung an unsere Kreatürlichkeit wieder. Stattdessen wird der Zustand des Abjekten in der Zerrissenheit des Subjekts geboren; im ständigen Widerstreit zwischen seinen Begierden und seiner Vernunft. ${ }^{4}$

Das Subjekt ist damit gezwungen, diese Zerrissenheit zu überwinden und sich selbst zu einer psychischen Einheit (vgl. dazu Kristeva 1982; Korsgaard 2009; Castoriadis 2012) wieder zusammenzufügen. Grundsätzlich kann damit der Prozess der Abjektion als die Herstellung von Subjektivität überhaupt verstanden werden, indem er die Einführung der primären existenziellen Grenze repräsentiert. Mit dieser Schöpfung wird erst dasjenige produziert, was sich innerhalb oder außerhalb dieser Grenzen verortet. Diese grundlegende Unterscheidung muss ihren Ursprung selbst aber nicht zwingend in einem Individuum haben. Ganz im Gegenteil: Mit Kristeva lässt sich beschreiben, dass es nur eine grundlegend psychobiologische Grenzziehung gibt - und das ist die Grenzziehung zwischen dem Selbst und dem Objekt (vgl. dazu Kristeva 1982). Alle anderen Grenzziehungen oder basale Unterscheidungen sind immer mit einer bestimmten kulturellen Logik oder Sprache verbunden, einem bestimmten symbolischen Raum - einer Kultur, Weltanschauung, Religion oder Lebensform.

Der Zustand des Abjekten bzw. die existenzielle Ungewissheit taucht insofern nur in der Irritation der Grenze auf, und diese Irritation spüren Subjekte nur, weil sie subjektiv oder kulturell noch keine klare oder eindeutige Möglichkeit gefunden haben, mit der Irritation umzugehen. Der Irritation der Grenzziehungen begegnen Subjekte in der Etablierung von Praktiken, die ständig reproduziert (aber auch ver-

\footnotetext{
4 An dieser Stelle wird auch verständlich, warum wir uns nach Kristeva von ,abjekten“ Dingen (also Dingen, die unseren Subjektstatus in Frage stellen) angezogen fühlen. Das Abjekt tritt uns eben nicht als ein konkretes Objekt gegenüber, sondern ist Ambivalenz oder Ungewissheit. Wir finden uns, weil der Zustand des Abjekt sich eben in der Gegenüberstellung von unserer Natürlichkeit und Vernunft verortet, in den ,abjekten“ Dingen wieder, die wir schließlich aus Ekel von uns entfernen wollen. Dass wir von bestimmten Dingen gleichzeitig abgestoßen und angezogen werden, ist kein Widerspruch. Stattdessen ist es Ausdruck der Frage, wer wir sind: Wir können uns in der Abstoßung und in der Anziehung wiederfinden.
} 
ändert und angepasst) werden müssen, um diese wieder re-etablieren zu können. Subjekte und soziale Gemeinschaften sind also insofern vor die Aufgabe gestellt, die Abjektion immer wieder zu meistern oder neu zu entwerfen und auch dem $\mathrm{Zu}$ stand der Ungewissheit, der existenziellen Angst und damit der existenziellen Krise mit neuen Formen bzw. Praktiken der Abjektion zu begegnen. Das Individuum, und schließlich auch die Gesellschaft, ist damit nicht nur vor die Aufgabe gestellt, sich immer wieder neu zu schöpfen, sondern kann genuin als menschliche Selbstschöpfung verstanden werden (vgl. dazu auch Castoriadis 1990).

\section{Der Preis der Freiheit: Ungewissheit und existenzielle Angst}

Im Verlust der Tatsache, sich nicht mehr nur auf seine Instinkte verlassen zu können, erfährt der Mensch, so Kant, einen Bruch. Er steht vor der Aufgabe, sich selbst zu schöpfen und findet sich in einem Zustand der Ungewissheit wieder: einer existenziellen Krise. Im Zustand der existenziellen Krise und der Angst, die damit einhergeht, verorte ich den Begriff des Abjekten.

Zerrissen - zwischen seiner Natürlichkeit und seiner Fähigkeit zur Vernunft steht das Subjekt vor der Aufgabe, sich wieder zusammenzusetzen. Und dies wird durch die Abjektion geleistet: Wir setzen uns im Handeln, in einem normativen Prinzip, in einer Grenzziehung wieder zusammen. Wir erschaffen uns selbst, in dem wir einen Willen, ein Handlungsprinzip im kantischen Sinne hervorbringen und uns mit diesem Willen identifizieren.

Der Begriff der Abjektion kann insofern an Kierkegaards (1992) und Heideggers (1979 [1927], 2006 [1929]) Philosophie der existenziellen Angst und der philosophischen Anthropologie Plessners und Gehlens (Fischer 2016) anschlussfähig gemacht werden und bietet damit eine psychoanalytische Ergänzung und Vervollständigung des Verständnisses einer grundlegenden Notwendigkeit des menschlichen Subjekts: sich selbst als ein, in der Zerrissenheit zwischen seiner Natürlichkeit und Vernunft lebendes Wesen, als Subjekt zu setzen.

Kristevas Begriffe des Abjekten und der Abjektion können aber nicht nur die Existenzphilosophie, sondern auch die soziologische Theoriebildung bereichern und jene Momente im Dasein von (kulturellen) Subjekten bestimmen und verständlich machen, die eine existenzielle Krise erfahren. Der Begriff der Abjektion bietet damit nicht nur die Möglichkeit, den Zustand kultureller Subjekte zu erfassen, sondern auch die Möglichkeit, den Umgang sozialer Subjekte zu beschreiben, die mit Phänomenen der Indifferenz oder Ambivalenz (Giesen 2011) konfrontiert sind. Und: der Begriff des Abjekten und der Abjektion kann für eine soziologische Theorie der Imagination bzw. des Imaginären und der Schöpfung von sozialem Sinn (Castoriadis 1990; vgl. Pfaller 2021) fruchtbar gemacht werden, welche zu erklären versucht, wie existenzielle soziale Krisen oder soziale Ungewissheiten von Subjekten bearbeitet werden - durch die Schöpfung einer sinnhaften Welt.

Förderung Der vorliegende Artikel ist entstanden im Rahmen des von der DFG geförderten Projekts „Das Imaginäre an den Grenzen des Sozialen“ (Projektnummer: 417783052).

Funding Open Access funding enabled and organized by Projekt DEAL. 
Open Access Dieser Artikel wird unter der Creative Commons Namensnennung 4.0 International Lizenz veröffentlicht, welche die Nutzung, Vervielfältigung, Bearbeitung, Verbreitung und Wiedergabe in jeglichem Medium und Format erlaubt, sofern Sie den/die ursprünglichen Autor(en) und die Quelle ordnungsgemäß nennen, einen Link zur Creative Commons Lizenz beifügen und angeben, ob Änderungen vorgenommen wurden.

Die in diesem Artikel enthaltenen Bilder und sonstiges Drittmaterial unterliegen ebenfalls der genannten Creative Commons Lizenz, sofern sich aus der Abbildungslegende nichts anderes ergibt. Sofern das betreffende Material nicht unter der genannten Creative Commons Lizenz steht und die betreffende Handlung nicht nach gesetzlichen Vorschriften erlaubt ist, ist für die oben aufgeführten Weiterverwendungen des Materials die Einwilligung des jeweiligen Rechteinhabers einzuholen.

Weitere Details zur Lizenz entnehmen Sie bitte der Lizenzinformation auf http://creativecommons.org/ licenses/by/4.0/deed.de.

\section{Literatur}

Arya, Rina. 2014. Abjection and Representation. Basingstoke: Palgrave Macmillan.

Bieri, Peter. 2007. Das Handwerk der Freiheit. Frankfurt a. M.: Suhrkamp.

Butler, Judith. 1993. Bodies that matter. New York/London: Routledge.

Castoriadis, Cornelius. 1990. Gesellschaft als imaginäre Institution. Frankfurt a. M.: Suhrkamp.

Castoriadis, Cornelius. 2012. Von der Monade zur Autonomie. In: Psychische Monade und Autonomes Subjekt. Hrsg. Michael Halfbrodt, Harald Wolf, Bd. 5, 47-76. Lich: Verlag Edition.

Fischer, Joachim. 2016. Philosophische Anthropologie: Eine Denkrichtung des 20. Jahrhunderts. Freiburg: Karl Alber Verlag.

Frankfurt, Harry. G. 2001. Willensfreiheit und der Begriff der Person. In Harry G. Frankfurt: Freiheit und Selbstbestimmung, Hrsg. Monika Betzler, Barbara Guckes, 65-83. Berlin: Akademie Verlag.

Freud, Sigmund. 1933. Neue Folge der Vorlesungen zur Einführung in die Psychoanalyse. Wien: Internationaler Psychoanalytischer Verlag.

Giesen, Bernhard. 2011. Zwischenlagen. Das Außerordentliche als Grund der sozialen Wirklichkeit. Weilerswist. Verlag: Velbrück.

Hegel, G.W.F. 1986. Wissenschaft der Logik, Bd. I. Werkausgabe, Bd. V. Frankfurt a. M.: Suhrkamp.

Heidegger, Martin 1979 [1927]. Sein und Zeit. Tübingen: Max Niemeyer Verlag.

Heidegger, Martin. 2006 [1929]. Was ist Metaphysik? Frankfurt a. M.: Vittorio Klostermann.

Kant, Immanuel. 1986. Kritik der praktischen Vernunft. Stuttgart: Reclam.

Kant, Immanuel. 1786. Band VIII: Abhandlungen nach 1781. In Mutmaßlicher Anfang der Menschheitsgeschichte. Gesammelte Schriften, Hrsg. Königlich-Preussischen Akademie der Wissenschaften zu Berlin, 107-125. 1902.

Kierkegaard, Sören. 1992. Der Begriff Angst. Stuttgart: Reclam.

Korsgaard, Christin M. 2009. Self-Constitution. Oxford: Oxford University Press.

Kristeva, Julia. 1982. Powers of Horror. New York: Columbia University Press.

Menninghaus, Winfried. 1999. Ekel: Theorie und Geschichte einer starken Empfindung. Frankfurt a. M.: Suhrkamp.

Pfaller, Larissa. 2021. Die dunkle Seite der Vorstellungskraft: Das Abjekt als Verworfenes im Imaginären. Österreichische Zeitschrift für Soziologie 46:301-319.

Taylor, Charles. 1983. Hegel. Frankfurt a. M.: Suhrkamp.

Taylor, Charles. 1996. Quellen des Selbst. Frankfurt a. M.: Suhrkamp.

Wittgenstein, Ludwig. 1984. Bemerkungen über die Farben, Über Gewißheit, Zettel, Vermischte Bemerkungen. Werkausgabe, Bd. VIII. Frankfurt a. M.: Suhrkamp. 